\title{
Le cancer à cellules germinales du testicule, élément constitutif du Syndrome de Dysgénésie Testiculaire : rôle des facteurs environnementaux et de la susceptibilité génétique
}

\author{
Ewa RAJPERT-DE MEYTS, Christina E. HOEl-HANSEN, Anne Marie OTTESEN, Lone \\ FRYDELUND-LARSEN, Mette HOLM, Niels E. SKAKKEBAEK \\ Department of Growth and Reproduction, Copenhagen University Hospital (Rigshospitalet), \\ Copenhagen, Denmark
}

RESUME

Durant les dernières décennies, le cancer du testicule à cellules germinales a augmenté dans de nombreux pays à travers le monde. Ces dernières années, l'attention a été attirée par les nombreuses recherches révélant des tendances négatives dans d'autres aspects de la santé reproductive masculine, comme une fréquence élevée et probablement en augmentation du testicule non descendu et de l'hypospadias, une qualité du sperme déclinante, et une demande apparemment croissante de reproduction assistée pour infécondité masculine. Le présent article résume les preuves qui sont en faveur d'un nouveau concept, à savoir que ces anomalies de la reproduction masculine pourraient constituer les signes d'une entité sous-jacente, le Syndrome de Dysgénésie Testiculaire (SDT). Ce syndrome, dû à des retards non spécifiques et à des aberrations du développement testiculaire précoce, pourrait devenir de plus en plus commun en raison de facteurs délétères de l'environnement et du mode de vie qui perturbent le développement gonadique. Les variations géographiques et ethniques de l'incidence des différentes formes de SDT peuvent s'expliquer soit par des différences d'exposition aux facteurs adverses, soit par des variations de la susceptibilité génétique à ces facteurs.

Mots clés : cancer du testicule, développement testiculaire, syndrome de dysgénésie testiculaire, perturbateurs environnementaux, susceptibilité génétique, reproduction masculine

\section{INTRODUCTION}

Le cancer du testicule à cellules germinales est un type de néoplasie relativement rare ; son impact négatif sur la population est toutefois sévère puisqu'il survient principalement chez les hommes jeunes et parce que son incidence est en augmentation dans la plupart des pays de l'Ouest $[32,2,29]$. Un nombre considérable de travaux récents indique que cette augmentation de l'incidence du cancer du testicule s'accompagne de tendances défavorables tout autant préoccupantes concernant différents autres aspects de la santé reproductive masculine $[60,33]$. Ces problèmes de santé comprennent un déclin rapporté de la concentration de spermatozoïdes dans nombre de régions de par le monde $[10,4,58,3,24]$, des incidences croissantes de cryptorchidie et d'hypospadias [11, 41, 61], ainsi qu'une proportion apparemment croissante de soit disant facteur masculin chez les couples infertiles traités dans les centres de fertilité.

Nous avons récemment avancé une hypothèse, à savoir que l'association observée du cancer du testicule avec d'autres désordres reproductifs n'est pas une coïncidence. Bien au contraire, tous ces désordres sont probablement les signes d'un développement testiculaire de pauvre qualité ; c'est pourquoi ils sont des éléments constitutifs du syndro-

\section{Correspondance :}

Ewa Rajpert-De Meyts, MD, PhD. - Department of Growth \& Reproduction, Rigshospitalet, GR-5064, 9 Blegdamsvej, DK2100 Copenhagen, Denmark - Fax +45 35455064 - Email erm@rh.dk 
me dénommé Syndrome de Dysgénésie Testiculaire (SDT) [53]. Notre hypothèse repose essentiellement sur la preuve de l'origine anténatale de la majorité des désordres reproductifs du mâle humain. Ce n'est pas seulement vrai pour les désordres congénitaux évidents, tels que l'hypospadias ou le testicule non descendu, mais aussi, de façon surprenante, pour des affections de l'âge adulte, comme le cancer du testicule ou l'infertilité masculine. La rapide vitesse de croissance des désordres reproductifs et le regroupement géographique des symptômes indiquent que des facteurs de l'environnement ou du mode de vie, plutôt que l'accumulation d'anomalies génomiques structurales, représentent les causes les plus probables du SDT. Ceci n'exclut pas que certaines aberrations ou certains polymorphismes génétiques puissent prédisposer à une augmentation des effets par des facteurs environnementaux ; de plus, dans de rares cas comme par exemple la néoplasie à cellules germinales associée aux états intersexués, ou le cancer testiculaire familial, les facteurs génétiques constituent le premier facteur causal. La présente revue discute les preuves suggérant qu'en cas de cancer sporadique du testicule, des facteurs de l'environnement semblent être les principaux responsables de l'augmentation épidémique observée au niveau de l'incidence.

\section{PREUVE EPIDEMIOLOGIQUE D'UNE ASSOCIATION DU CANCER DU TESTICU- LE AVEC D'AUTRES DESORDRES REPRO- DUCTIFS, ET DE LEUR ORIGINE DEVE- LOPPEMENTALE}

Le cancer du testicule à cellules germinales des adolescents et des adultes jeunes provient de cellules germinales présentant un carcinome in situ (CIS) dont on présume qu'elles dérivent de cellules germinales primordiales/gonocytes ayant échappé à une différenciation normale $[52,44]$. Les cellules ayant un CIS ressemblent étroitement aux gonocytes, leur morphologie est très semblable, et les deux types cellulaires expriment de nombreux marqueurs immunochimiques communs [voir revue dans 45].

Il est bien établi que de rares anomalies génétiques qui entraînent une dysgénésie testiculaire (par exemple $45 \mathrm{X} / 46 \mathrm{XY}$ et l'insensibilité aux androgènes) sont associées à un risque élevé de cancer du testicule, souvent en combinaison avec le testicule non descendu et l'hypospadias [1, $49,50]$. De la même façon, il est établi par plusieurs études que des hommes avec un testicule non descendu et/ou un hypospadias sont de façon significative sur-représentés parmi les patients ayant un cancer du testicule $[5,18]$. On sait aussi que le parenchyme testiculaire adjacent aux tumeurs à cellules germinales [55] et le testicule controlatéral d'hommes avec cancer unilatéral du testicule [7] sont souvent anormalement développés. Les signes de faible développement sont constitués de tubules ne contenant que des cellules de Sertoli, qui sont elles-mêmes souvent peu différenciées, d'un arrêt de la spermatogénèse, de microcalcifications, et aussi, dans environ 5 à $7 \%$ des cas, de CIS (Figure 1). Les données systématiques sont absentes ; c'est pourquoi nous avons récemment réalisé de grosses études de cohorte, portant sur les signes histologiques et cliniques de dysgénésie testiculaire dans les biopsies controlatérales : les résultats confirment l'association du cancer du testicule à cellules germinales avec la dysgénésie testiculaire (HoeiHansen et al., manuscrit en préparation ; Holm et al., manuscrit en préparation). Pour ce qui est de la cryptorchidie, l'appartenance de cette anomalie au SDT ne fait aucun doute. Le testicule non descendu est toujours associé à un
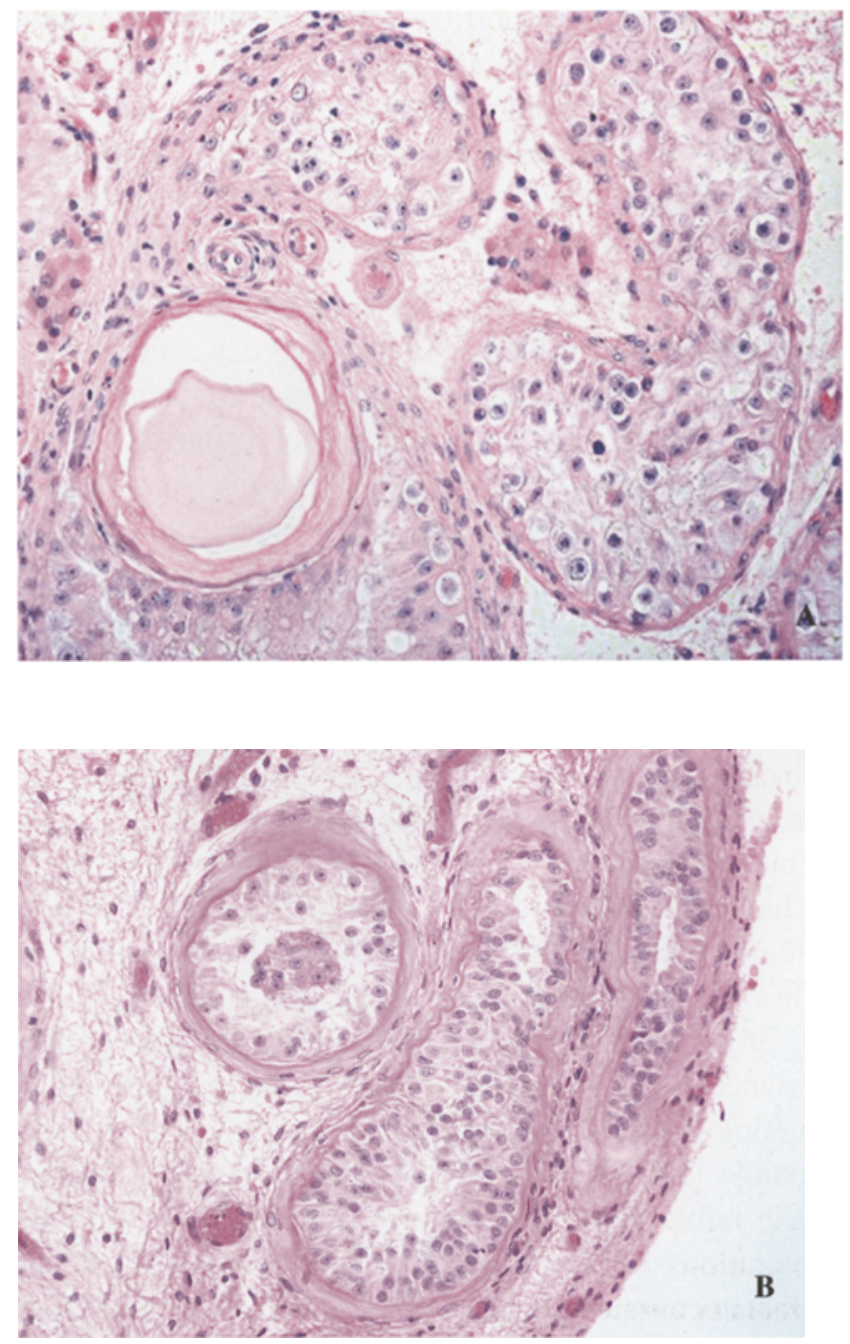

Figure 1 : Exemple de dysgénésie testiculaire en association avec une tumeur testiculaire à cellule germinale.

A. La photographie montre des tubules contenant des cellules avec un carcinome in situ (CIS) et une volumineuse microcalcification (microlithe).

B. Tubules indifférenciés, contenant des cellules de Sertoli immatures, trouvés dans le parenchyme testiculaire adjacent à une tumeur à cellule germinale. 
certain degré de maldéveloppement des tubules séminifères, incluant des tubules avec uniquement des cellules de Sertoli et des arrêts de la spermatogénèse [54, 19]. Le testicule non descendu peut aussi renfermer des microcalcifications et des groupes de tubules indifférenciés.

Ce n'est pas seulement l'histologie, mais aussi la fonction du testicule qui est altérée lors de la néoplasie à cellule germinale, même avant qu'une tumeur ouverte ne se soit développée. Les concentrations de spermatozoïdes chez les hommes avec cancer du testicule sont souvent très basses, beaucoup plus basses qu'on l'attendrait chez un homme avec un seul testicule fonctionnel [43]. De plus, plusieurs études récentes ont montré que les hommes avec un cancer du testicule avaient une fécondité significativement réduite, avec une moindre proportion d'enfants mâles (diminution du sex ratio) et des caractéristiques spermatiques anormales avant le développement de leur tumeur [35, 21, 22, 15]. Les résultats de ces études constituent une preuve solide étayant notre hypothèse que la détérioration de la spermatogénèse et le cancer du testicule sont étiologiquement liés.

La présomption que des facteurs habituels du développement soient responsables des évolutions des cancers du testicule, des malformations génitales et de la qualité de la spermatogénèse, est en outre corroborée par des preuves épidémiologiques. Les plus parlantes sont constituées par les découvertes d'un effet cohorte de naissance dans l'incidence du cancer du testicule, découvertes basées sur les observations intrigantes que les hommes scandinaves nés durant la guerre ont une fréquence du cancer du testicule à l'âge adulte plus basse qu'attendue à partir de l'incidence globale de la maladie $[32,6]$. Comme rappelé plus haut, l'association de la cryptorchidie avec le cancer du testicule est bien documentée $[9,31,18]$. Le testicule non descendu est habituellement présent à la naissance, c'est pourquoi cette condition est, par définition, d'origine fœtale et cette malformation s'accompagne d'un faible poids de naissance $[34,36]$. Une forte association semblable entre poids de naissance et cancer du testicule est en accord avec une liaison étiologique bien documentée entre ce cancer et la cryptorchidie [12, 34]. De plus, des études récentes indiquent que le faible poids de naissance est associé avec de bases numérations de spermatozoïdes [16]. Enfin, des facteurs périnataux auraient un rôle dans les tendances rapportées du cancer du testicule, des malformations génitales et de la qualité du sperme, comme cela a été suggéré par plusieurs études menées en Europe et en Amérique du Nord [4, 34, $65,24]$.

Les observations épidémiologiques fournissent la preuve d'une association géographique de divers constituants du SDT. Par exemple, en Finlande, les taux de cancer du testicule, de testicule non descendu et d'hypospadias sont beaucoup plus bas qu'au Danemark. Il en est de même pour la qualité du sperme qui est bien meilleure en Finlande qu'au Danemark. En outre, les taux de chacune de ces conditions croissent de façon synchrone dans les deux pays. En conséquence, le SDT pourrait être plus commun qu'il apparaît au premier abord car des formes modérées peuvent ne se présenter qu'avec une légère détérioration de la spermatogénèse. Nos études sur la qualité du sperme d'hommes jeunes issus de la population générale danoise suggèrent que la forme modérée du SDT, qui inclue une pauvre qualité spermatique (concentration de spermatozoïdes $<20$ millions/ml), est plutôt habituelle [3] et pourrait commencer à avoir un impact sur la fécondité de la population danoise [23]. A l'autre extrémité de l'échelle, les formes les plus sévères du SDT, incluant les états intersexués et le cancer du testicule, apparaissent être relativement rares.

\section{ETIOLOGIE DU CANCER DU TESTICU- LE ET DU SDT: ENVIRONNEMENT OU PREDISPOSITION GENETIQUE ?}

Le SDT peut être dû à des facteurs génétiques ou environnementaux, ou à une combinaison des deux. Le développement de la génétique moléculaire moderne a étendu la compréhension de la génétique des désordres endocrines, y compris la dysgénésie testiculaire, qui peuvent être causés par une multitude de défauts génétiques [voir 20 et 42 pour une revue]. Cependant, en l'état actuel de nos connaissances, chez la majorité des hommes avec des malformations génitales et un cancer du testicule, aucune anomalie chromosomique ou génétique n'a pu être démontrée en dehors du tissu d'une tumeur ouverte [28, 40, Ottesen et al., en préparation ; Frydelund-Larsen et al., en préparation]. Les cas de tumeur familiale sont extrêmement rares et aucune aberration spécifique n'a encore été retrouvée dans ces cas. Les études dans ces familles rares n'ont identifié jusqu'à maintenant qu'une seule zone de susceptibilité potentielle, Xq27, qui est commune à la fois à la cryptorchidie et au cancer du testicule [48]. Pour ce qui est des cas sporadiques, la recherche de mutations spécifiques a été jusqu'alors infructueuse, sauf pour quelques rares mutations trouvées dans l'ADN tumorale d'un petit nombre de cas de séminomes [8].

La preuve épidémiologique est la plus compatible avec le rôle étiologique premier des facteurs de l'environnement ou du mode de vie. Les hormones synthétiques et les perturbateurs endocriniens à activité oestrogénique et anti-androgénique, dont l'utilisation est largement répandue, ne peuvent être exclus en tant que potentiels facteurs défavorables, puisque le développement du système reproducteur masculin est exquisément sensible aux hormones sexuelles stéroïdiennes $[51,60]$. Le développement de la gonade est, pour une grande part, régulé par les hormones sexuelles et quelque soit le sexe génétique, il peut se développer soit 
comme testicule, soit comme ovaire, ou comme quelque chose d'intermédiaire, si cette régulation est perturbée. Si la différenciation des cellules de Sertoli et de Leydig, qui produisent des hormones stéroïdiennes et leur répondent directement, est perturbée, la prolifération des cellules germinales sera défectueuse (Figure 2). Le résultat final en sera généralement une anomalie génitale et/ou une cryptorchidie, suivie plus tard dans la vie par des problèmes de fécondité et, dans certains cas, de cancer du testicule. Même si les symptômes détectables cliniquement apparaissent après la naissance, la cause sous-jacente en est une dysgénésie testiculaire irréversible lors du développement fotal précoce.

Toutefois, l'impact potentiel des perturbateurs endocriniens n'a pas été prouvé chez les humains, excepté pour certains effets du DES sur le fotus humain [57].
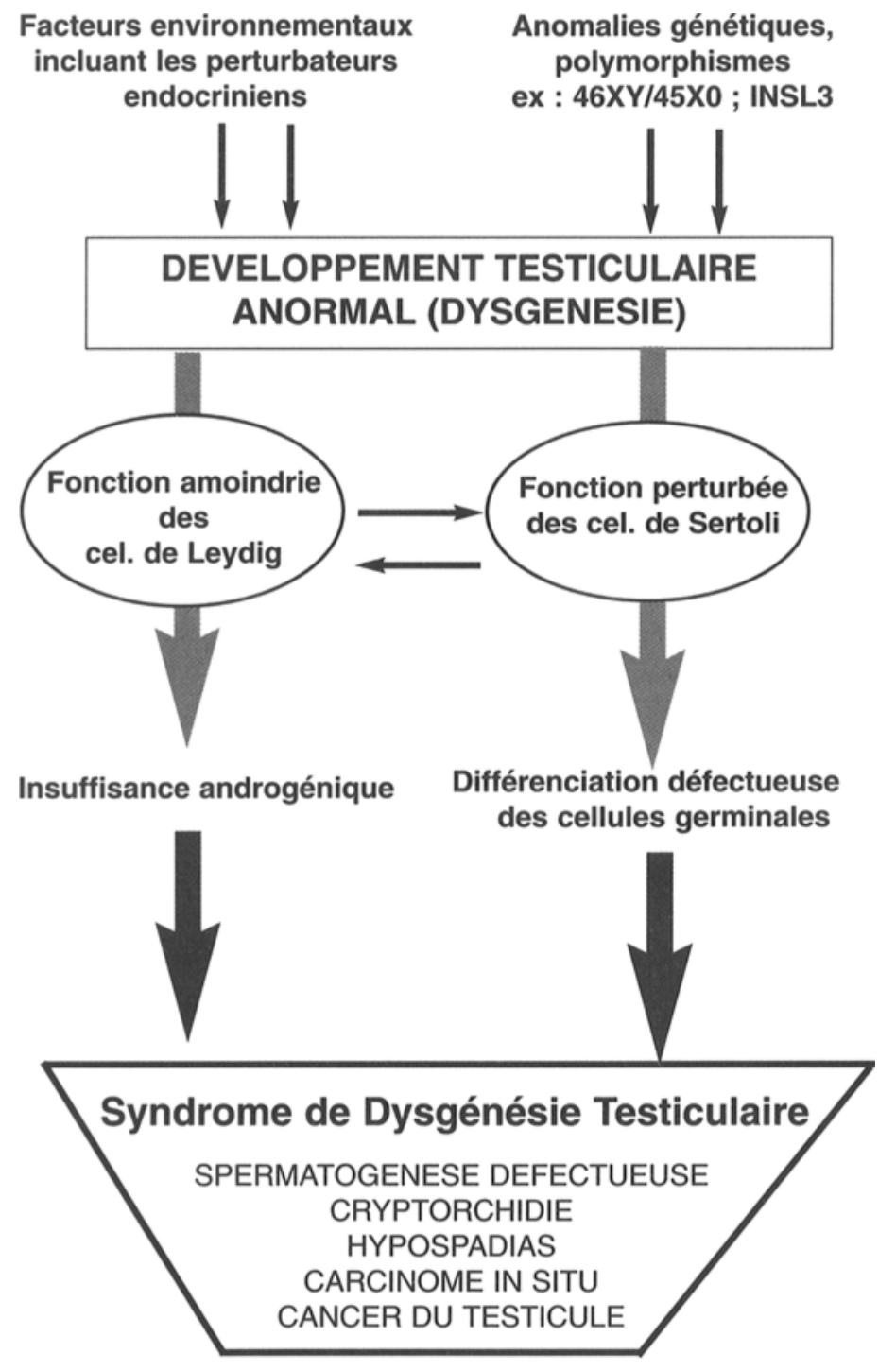

Figure 2 : Représentation schématique des liens pathogénétiques entre les constituants et les manifestations cliniques du syndrome de dysgénésie testiculaire (SDT). Noter que, alors que l'incidence globale du SDT décroût avec le dégré de sévérité, l'incidence relative du cancer du testicule augmente. Adapté de Sakkebaek et al. [53].
Les études épidémiologiques rapportent cependant un risque accru de malformations génitales chez les enfants de travailleurs exposés professionnellement aux pesticides [64] ainsi qu'une concentration importante de cryptorchidie dans les régions d'agriculture intensive [17]. Les arguments en faveur de l'hypothèse des perturbateurs endocriniens proviennent des observations faites chez l'animal. Il existe une profusion de données qui indiquent que chez des animaux mâles, exposés in utero ou en période périnatale à des œstrogènes et/ou des anti-androgènes exogènes, se développent des atrophies testiculaires, des cryptorchidies, des hypospadias, ou même des états intersexués $[62,63,25$, 30]. De façon intéressante, de récentes études ont démontré que le gène Insl3, qui est impliqué dans la migration testiculaire chez les rongeurs [37], est très certainement régulé par les œstrogènes, puisque une exposition prénatale suffisante pour entrainer l'apparition d'une cryptorchidie expérimentale entraîne une régulation négative de ce gène [14, 38]. Toutefois, l'extrapolation des observations animales aux humains est très difficile, tout particulièrement parce que la même exposition entraîne quelques fois des effets immensément différents chez des animaux de mêmes espèces. Une explication possible pourrait être l'existence de différences dans le bagage génétique d'animaux de laboratoire consanguins, différences qui affecteraient leur susceptibilité aux hormones exogènes.

L'observation d'effets très largement différents des hormones chez des lignées différentes de souris suggère que la diversité génétique puisse aussi être responsable de différences ethniques en ce qui concerne la prévalence des désordres reproductifs chez l'homme, par exemple la différence d'incidence du cancer du testicule entre les Européens et les Africains. Il est plausible que certains gènes, qui sont impliqués dans la régulation hormonale du développement testiculaire, puissent contenir des séquences polymorphiques qui altèreraient légèrement la sensibilité aux hormones naturelles ou synthétiques. Toutefois, l'association d'un polymorphisme du candidat le plus évident, le gène du récepteur aux androgènes, avec l'infécondité, reste discutée $[13,26]$. De plus, aucune association du polymorphisme du gène du récepteur aux androgènes n'a été trouvée avec le cancer du testicule [39,47]. Plusieurs études récentes ont identifié des polymorphismes du gène humain INSL3, mutations qui sont extrêmement rares [26, 27, 59]. Bien que les polymorphismes mentionnés ici n'aient pas été fermement associés avec des altérations chez l'homme, il n'est pas déraisonnable de spéculer sur la possibilité que les polymorphismes d'autres gènes puissent les rendre plus enclins à des modifications de la régulation transcriptionnelle par les hormones du développement du système reproducteur. 


\section{CONCLUSIONS ET PERSPECTIVES FUTURES}

Des preuves s'accumulent allant des observations cliniques individuelles aux larges études épidémiologiques, qui indiquent une augmentation synchronisée chez l'homme de l'incidence des problèmes reproductifs tels que le cancer du testicule, les anomalies génitales, une qualité réduite du sperme et une hypofertilité. Des associations chronologiques et géographiques suggèrent fortement l'existence d'un lien pathogénique commun. D'après notre hypothèse, ces désordres sont liés à un pauvre développement du testicule, et nous avons nommé le phénotype qui en résulte le Syndrome de Dysgénésie Testiculaire (SDT). Des explorations expérimentales chez l'animal et des études épidémiologiques suggèrent que le SDT résulte probablement d'une rupture de la programmation embryonnaire et du développement gonadique pendant la vie fotale. Comme l'accroissement de l'incidence des différents symptômes du SDT sont survenus rapidement en quelques générations, l'impact étiologique de facteurs défavorables de l'environnement agissant sur un fond de susceptibilité génétique doit être pris en considération.

\section{Remerciements :}

Les travaux du Groupe du Département de Croissance et Reproduction, Université de Copenhague, résumés dans cette revue, ont fait l'objet de subventions des Danish Cancer Society, Danish Medical Research Council, Andersen's Fundation, de l'Union Européenne et du Council of Copenhagen University Hospitals.

\section{REFERENCES}

1. AARSKOG D. : Clinical and cytogenetic studies in hypospadias. Acta Paediatr. Scand., 1970, Suppl. 203 : 1-62.

2 ADAMI H., BERGSTRÖM R., MÖHNER M. et al. : Testicular cancer in nine northern European countries. Int. J. Cancer, 1994, $59: 33-38$.

3. ANDERSEN A.G., JENSEN T.K., CARLSEN E. et al. : High frequency of sub-optimal semen quality in an unselected population of young men. Hum. Reprod., 2000, 15 : 366-372.

4 AUGER J., KUNSTMANN J.M., CZYGLIK F., JOUANNET P.: Decline in semen quality among fertile men in Paris during the past 20 years. N. Engl. J. Med., 1995, 332 : 281-285.

5. BATATA M.A., CHU F.C.H., HILARIS B.S., WHITMORE W.F., GOLBEY R.B. : Testicular cancer in cryptorchids. Cancer, 1982, $49: 1023-1030$.

6. BERGSTRÖM R., ADAMI H., MOHNER M. et al. : Increase in testicular cancer incidence in six European countries : a birth cohort phenomenon. J. Natl. Cancer Inst., 1996, 88 : 727-733.

7. BERTHELSEN J.G., SKAKKEBAEK N.E. : Gonadal function in men with testis cancer. Fertil. Steril., 1983, $39: 68-75$.
8. BOURAS M., TABONE E., BERTHOLON J. et al. : A novel SMAD4 gene mutation in seminoma germ cell tumors. Cancer Res., 2000, 60 : 922-928.

9. CAMPBELL H.E. : Incidence of the malignant growth of the undescended testicle. A critical and statistical study. Arch. Surg., $1942,44: 353-369$.

10. CARLSEN E., GIWERCMAN A., KEIDING N., SKAKKEBAEK N.E. : Evidence for decreasing quality of semen during past 50 years. Br. Med. J., 1992, 305 : 609-613.

11. CHILVERS C., PIKE M.C., FORMAN D., FOGELMAN K., WADSWORTH M.E.J. : Apparent doubling of frequency of undescended testis in England and Wales in 1962-81. Lancet, 1984, ii : 330-332.

12. DEPUE R.H., PIKE M.C., HENDERSON B.E. : Birth weight and the risk of testicular cancer. J. Natl. Cancer. Inst., 1986, 77 : 829830.

13. DOWSING A.T., YOUNG E.L., CLARK M., MCLACHLAN R.I., DE KRETSER D.M., TROUNSON A.O. : Linkage between male infertility and trinucleotide repeat expansion in the androgen-receptor gene. Lancet, 1999, 354 : 640-643.

14. EMMEN J.M.A., MCLUSKEY A., ADHAM I, et al. : Involvement of insulin-like factor 3 (Insl3) in diethylstilbestrolinduced cryptorchidism. Endocrinology 2000, $141: 846-849$.

15. FOSSÅ S.D., KRAVDAL $\varnothing$. : Fertility in Norwegian testicular cancer patients. Brit. J. Cancer, 2000, 82 : 737-741.

16. FRANCOIS I., DE ZEGHER F., SPIESSENS C., D'HOOGE T., VANDERSCHUEREN D. : Low birth weight and subsequent male subfertility. Pediatr. Res., 1997, 42 : 899-901.

17. GARCIA-RODRIGUEZ J., GARCIA-MARTIN M., NOGUERAS-OCANA $M$. et al. : Exposure to pesticides and cryptorchidism : geographical evidence of a possible association. Environ. Health Perspect., 1996, 104 : 1090-1095.

18. GIWERCMAN A., BRUUN E., FRIMODT-MOLLER C., SKAKKEBAEK N.E. : Prevalence of carcinoma in situ and other histopathological abnormalities in testis of men with a history of cryptorchidism. J. Urol., 1989, 142 : 998-1001.

19. HUFF D.S., HADZISELIMOVIC F., SNYDER H.M., BLYTHE B., DUCKETT J.W. : Histologic maldevelopment of unilaterally cryptorchid testis and their descended partners. Eur. J. Pediatr., 1993, 152 (Suppl. 2) : 11-14.

20. HUGHES I.A. : Sexual Differentiation. In : Baillière's Clinical Endocrinology and Metabolism. London, Baillière Tindall,1998 : 198.

21. JACOBSEN R., BOSTOFTE E., ENGHOLM G., HANSEN J., SKAKKEBAEK N.E., MØLLER H. Fertility and offspring sex ratio of men who develop testicular cancer : a record linkage study. Hum. Reprod., 2000, 15 : 1958-1961.

22. JACOBSEN R., BOSTOFTE E., ENGHOLM G. et al. : Risk of testicular cancer in men with abnormal semen characteristics : cohort study. Br. Med. J., 2000, 321 : 789-792.

23. JENSEN T.K., CARLSEN E., JØRGENSEN N. E. et al. : Hypothesis : Poor semen quality may contribute to recent decline in fertility rates. Hum. Reprod., 2002, $17: 1437-1440$.

24. JØRGENSEN N., ANDERSEN A.-G., EUSTACHE F. et al. : Regional differences in semen quality in Europe. Hum. Reprod., 2001, $16: 1012-1019$.

25. KELCE W.R., STONE C.R., LAWS S.C., GRAY L.E., WILSON E.M. : The persistent DDT metabolite p,p'-DDE is a potent androgen receptor antagonist. Nature, 1995, $375: 581-585$. 
26. KRAUSZ C., QUINTANA-MURCI L., FELLOUS M., SIFFROI J.-P., MCELREAVEY K. : Absence of mutations involving the INSL3 gene in human idiopathic cryptorchidism. Mol. Hum. Reprod., 2000, 6 : 298-302.

27. LIM H.N., RAJPERT-DE MEYTS E., SKAKKEBAEK N.E., HAWKINS J.R., HUGHES I.A. : Genetic analysis of the INSL3 gene in patients with maldescent of the testis. Euro. Endocrinol., 2001, 144 : 129-37.

28. McELREAVEY K. : Y-a-t-il des gènes du cancer du testicule ? Andrologie, 2000, 10:171-180.

29. MC KIERNAN J.M., GOLUBOFF E.T., LIBERSON G.L., GOLDEN R., FISCH H. : Rising risk of testicular cancer by birth cohort in the United States from 1973 to 1995. J. Urol., 1999, 162: 361-363.

30. MCLACHLAN J.A., NEWBOLD R.R., BUROW M.E., LI S.F. : From malformations to molecular mechanisms in the male : three decades of research on endocrine disrupters. APMIS 2001, 109 : 263-272.

31. MORRISON A.S. : Cryptorchidism, hernia and cancer of the testis. J. Natl Cancer Inst., 1976, 56 : 731-733.

32 MØLLER H. : Clues to aetiology of testicular germ cell tumours from descriptive epidemiology. Eur. Urol., 1993, 23 : 8-15.

33. MØLLER $H$. : Trends in sex-ratio, testicular cancer and male reproductive hazards : Are they connected ? APMIS 1998, 106 : 232-239.

34. MØLLER H., SKAKKEBAEK N.E. : Testicular cancer and cryptorchidism in relation to prenatal factors : case-control studies in Denmark. Cancer Causes Control, 1997, 8 : 904-912.

35. MØLLER H., SKAKKEBAEK N.E. : Risk of testicular cancer in subfertile men : case-control study. Br. Med. J., 1999, 318 : 559562.

36. MORLEY R., LUCAS A. : Undescended testis in low birth weight infants. Br. Med. J., 1987, $295: 753$.

37. NEF S., PARADA L.F. : Cryptorchidism in mice mutant for Insl3. Nat. Genet., 1999, 22 : 295-299.

38. NEF S., SHIPMAN T., PARADA L.F. : A molecular basis for estrogen-induced cryptorchidism. Dev. Biol., 2000, 224 : 354361.

39. ONO Y., RAJPERT-DE MEYTS E., GUELLAËN G., BULLE F.: Sporadic testicular germ cell cancers do not exhibit specific alteration in CAG/CTG repeats containing genes expressed in human testis. Oncogene, 2001, $50: 5548-5553$.

40. OTTESEN A.M., KIRCHHOFF M., RAJPERT-DE MEYTS E. et al. : Detection of chromosomal aberrations in seminomatous germ cell tumours using comparative genomic hybridization. Genes Chromosomes Cancer, 1997, $20: 412-418$.

41. PAULOZZI L.J., ERICKSON J.D., JACKSON R.J. : Hypospadias trends in two US surveillance systems. Pediatrics, 1997, $100: 831-834$.

42. PINSKY L., ERICKSON R.P., SCHIMKE R.N. : Genetic Disorders of Human Sexual Development. New York, Oxford University Press, 1999 : 410.

43. PETERSEN P.M., SKAKKEBAEK N.E., GIWERCMAN A. : Gonadal function in men with testicular cancer : Biological and clinical aspects. APMIS 1998, 106 : 24-36.

44. RAJPERT-DE MEYTS E., JØRGENSEN N., BRØNDUMNIELSEN K., MÜLLER J., SKAKKEBAEK N.E. : Developmental arrest of germ cells in the pathogenesis of germ cell neoplasia. APMIS 1998, $106:$ 198-206.

45. RAJPERT-DE MEYTS E., GIWERCMAN A., SKAKKEBAEK N.E. : Le carcinome in situ du testicule - un précurseur du cancer à cellule germinale. Revue de la pathogenèse et aspects cliniques. Andrologie, 2000, $10: 181-193$.

46. RAJPERT-DE MEYTS E., LEFFERS H., PETERSEN H.J. et al.: The CAG repeat length in the androgen receptor gene and reproductive variables in fertile and infertile men. Lancet, 2002, 359 : 44-46.

47. RAJPERT-DE MEYTS E., LEFFERS H., DAUGAARD G. et al: Analysis of the polymorphic CAG repeat length in the androgen receptor gene in patients with testicular germ cell cancer. Int. J. Cancer, 2002, in press.

48. RAPLEY E.A., CROCKFORD G.P., TEARE D. et al. : Localization to Xq27 of a susceptibility gene for testicular germcell tumours. Nature Genetics, 2000, $24:$ 197-200.

49. SAVAGE M.O., LOWE D.G. : Gonadal neoplasia and abnormal sexual differentiation. Clin. Endocrinol., 1990, 32 : 519-533.

50. SCULLY R.E. : Neoplasia associated with anomalous sexual development and abnormal sex chromosomes. Ped. Adolesc. Endocrinol., 1981, 8 : 203-217.

51. SHARPE R.M., SKAKKEBAEK N.E. : Are oestrogens involved in falling sperm counts and disorders of the male reproductive tract ? Lancet, 1993, $341: 1392-1395$.

52. SKAKKEBAEK N.E., BERTHELSEN J.G., GIWERCMAN A., MÜLLER J. : Carcinoma-in-situ of the testis : possible origin from gonocytes and precursor of all types of germ cell tumours except spermatocytoma. Int. J. Androl., 1987, 10 : 19-28.

53. SKAKKEBAEK N.E., RAJPERT-DE MEYTS E., MAIN K.M. : Testicular dysgenesis syndrome : an increasingly common developmental disorder with environmental factors. Hum. Reprod., 2001, 16: 972-978.

54. SOHVAL A.R. : Testicular dysgenesis as an etiologic factor in cryptorchidism. J. Urol., 1954, $72: 693-702$.

55. SOHVAL A.R. : Testicular dysgenesis in relation to neoplasm of the testicle. J. Urol., 1956, $75: 285-291$.

56. SPEAROW J.L., DOEMENY P., SERA R., LEFFLER R., BARKLEY M. : Genetic variation in susceptibility to endocrine disruption by estrogen in mice. Science, 1999, $285: 1259-1261$.

57. SWAN S.H. : Intrauterine exposure to diethylstilbestrol : longterm effects in humans. APMIS 2000, $108: 793-804$.

58. SWAN S.H., ELKIN E.P., FENSTER L. : Have sperm densities declined? A reanalysis of global trend data. Environ. Health Perspect., 1997, 105 : 1228-1232.

59. TOMBOC M., LEE P.A., MITWALLY M.F., SCHNECK F.X., BELLINGER M., WITCHEL S.F. : Insulin-like 3/relaxin-like factor gene mutations are associated with cryptorchidism. J. Clin. Endocrinol. Metab, 2000, 85 : 4013-4018.

60. TOPPARI J., LARSEN J.C., CHRISTIANSEN P. et al. : Male reproductive health and environmental xenoestrogens. Environ. Health Perspect., 1996, 104 (suppl.4) : 741-803.

61. TOPPARI J., KALEVA M., VIRTANEN H.E. : Trends in the incidence of cryptorchidism and hypospadias, and methodological limitations of registry-based data. Hum. Reprod. Update, 2001, 7: 282-286.

62. VIGUIER-MARTINEZ M.C., HOCHEREAU DE REVIERS M.T., BARENTON B., PERREAU C. : Effect of a non-steroidal antiandrogen, flutamide, on the hypothalamo-pituitary axis, geni- 
tal tract and testis in growing rats: endocrinological and histological data. Acta Endocrinologica, 1983, 102 : 299-306.

63. WALKER A.H., BERNSTEIN L., WARREN D.W., WARNER N.E., ZHENG X., HENDERSON B.E. : The effect of in utero ethinyl oestradiol exposure on the risk of cryptorchid testis and testicular teratomas in mice. Br. J. Cancer, 1990, 62 : 599-602.

64. WEIDNER I.S., MøLLER H., JENSEN T.K., SKAKKEBAEK N.E. : Cryptorchidism and hypospadias in sons of gardeners and farmers. Environ. Health Perspect., 1998, 106 : 793-796.

65. WEIR H.K., MARRETT L.D., KREIGER N., DARLINGTON G.A., SUGAR L. : Pre-natal and peri-natal exposures and risk of testicular germ-cell cancer. Int. J. Cancer, 2000, 87 : 438-443.

\author{
ABSTRACT \\ Germ Cell Cancer and Testicular Dysgenesis \\ Syndrome
}

\begin{abstract}
E. RAJPERT-DE MEYTS, C.E. HOEI-HANSEN, Anne Marie OTTESEN, Lone FRYDELUND-LARSEN, Mette HOLM, Niels E. SKAKKEBAEK
\end{abstract}

The incidence of testicular germ cell cancer has been increasing over recent decades in many countries of the world. Many studies over recent years have reported adverse trends in other aspects of male reproductive health, such as high and possibly increasing frequencies of undescended testis and hypospadias, declining semen quality, and an apparently growing demand for assisted reproduction due to male infertility. This article summarises the available evidence supporting a new concept that these male reproductive abnormalities may be signs of a single underlying entity : testicular dysgenesis syndrome (TDS). This syndrome, caused by nonspecific delays and aberrations of early testicular development, may be increasingly common because of deteriorating environmental and life-style factors that impair gonadal development. Geographical and ethnic differences in the incidence of various forms of TDS could be explained either by differences in exposure to adverse factors or by differences in genetic susceptibility to these factors.

Key words: testicular cancer, testicular development, testicular dysgenesis syndrome, environmental disrupters, infertility, genetic susceptibility, male reproduction 